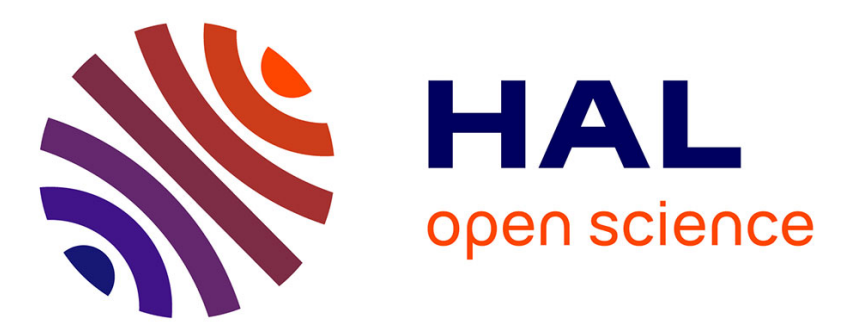

\title{
Exact solution to Duffing equation and the pendulum equation
}

\author{
Alvaro H. Salas, Jairo E. Castillo H.
}

\section{To cite this version:}

Alvaro H. Salas, Jairo E. Castillo H.. Exact solution to Duffing equation and the pendulum equation. Applied Mathematical Sciences, 2014, 8 (176), pp. 8781-8789. 10.12988/ams.2014.44243 . hal01356787

\section{HAL Id: hal-01356787 \\ https://hal.science/hal-01356787}

Submitted on 26 Aug 2016

HAL is a multi-disciplinary open access archive for the deposit and dissemination of scientific research documents, whether they are published or not. The documents may come from teaching and research institutions in France or abroad, or from public or private research centers.
L'archive ouverte pluridisciplinaire HAL, est destinée au dépôt et à la diffusion de documents scientifiques de niveau recherche, publiés ou non, émanant des établissements d'enseignement et de recherche français ou étrangers, des laboratoires publics ou privés. 
Applied Mathematical Sciences, Vol. 8, 2014, no. 176, 8781 - 8789

HIKARI Ltd, www.m-hikari.com

http://dx.doi.org/10.12988/ams.2014.44243

\title{
Exact Solution to Duffing Equation and the Pendulum Equation
}

\author{
Alvaro H. Salas \\ Universidad de Caldas, Departamento de Matemáticas \\ Universidad Nacional de Colombia Sede Manizales-Nubia Campus \\ FIZMAKO Research Group \\ Jairo E. Castillo H. \\ Universidad Distrital Francisco José de Caldas, Bogotá-Colombia \\ FIZMAKO Research Group \\ Copyright (c) 2014 Alvaro H. Salas and Jairo E. Castillo H.. This is an open access article \\ distributed under the Creative Commons Attribution License, which permits unrestricted \\ use, distribution, and reproduction in any medium, provided the original work is properly
} cited.

\begin{abstract}
In this work we obtain an exact solution to cubic Duffing oscillator equation with initial conditions and bounded periodic solutions. This solution is expressed in terms of the Jacobi elliptic function cn. In particular, we may apply the exact solution in the study of the cubic nonlinear Schrodinger equation, which is reduced to a cubic Duffing oscillator equation by means of a traveling wave transformation.
\end{abstract}

Keywords: Duffing equation, cubic Duffing oscillator equation, analytic solution, Jacobi elliptic functions, cubic nonlinear Schroedinger equation

\section{Introduction}

Serious studies of forced nonlinear oscillators appeared early in the 20th century when Georg Duffing (1918) examined mechanical systems with nonlinear 
restoring forces and Balthasar van der Pol studied electrical systems with nonlinear damping. Subsequently, any equation of the form

$$
\ddot{x}+b \dot{x}+f(x)=F \sin \omega t
$$

was called Duffing's equation, the nonlinearity $f(x)$ often being polynomial, usually cubic. Here, $\dot{x}$ represents the time derivative $\frac{d x}{d t}$. Linear resonance has $f(x)=\alpha x(c>0)$ and Duffing's extension models many mechanical and electrical phenomena. For a cubic nonlinearity $f(x)=\alpha x+\beta x^{3}$, if $\beta>0$, the system is hardening. When $\beta<0$ we deal with a softening system. In this work our objective is to derive the exact solution to Duffing equation. We use this solution to obtain the solution to pendulum equation.

\section{General solution to Duffing Equation}

We are going to find the general solution to Dufiing's equation

$$
\ddot{x}+\alpha x+\beta x^{3}=0
$$

in terms of the Jacobi elliptic function cn. This function is defined as follows:

$$
\operatorname{cn}(t, m)=\cos \phi, \text { where } t=\int_{0}^{\phi} \frac{d \theta}{\sqrt{1-m^{2} \sin ^{2} \theta}} .
$$

There are other two important elliptic Jacobi functions : sn and dn. They are defined by

$$
\operatorname{sn}(t, m)=\sin \phi \text { and } \operatorname{dn}(t, k)=\sqrt{1-m^{2} \sin ^{2} \phi} .
$$

The number $m(0<m<1)$ is called elliptic modulus and the number $\phi$ is called Jacobi amplitude and it is denoted by $\operatorname{am}(t, m)$. Thus,

$$
\phi=\operatorname{am}(t, m), \sin (\phi)=\sin (\operatorname{am}(t, m))=\operatorname{sn}(t, m)
$$

Following identities meet :

$$
\begin{gathered}
\operatorname{sn}^{2}(t, m)+\operatorname{cn}^{2}(t, m)=1, \operatorname{dn}^{2}(t, m)=1-m^{2} \operatorname{sn}^{2}(t, m) \\
\lim _{m \rightarrow 0} \operatorname{sn}(t, m)=\sin t, \lim _{m \rightarrow 0} \operatorname{cn}(t, m)=\cos t, \lim _{m \rightarrow 0} \operatorname{dn}(t, m)=1 . \\
\lim _{m \rightarrow 1} \operatorname{sn}(t, m)=\tanh t, \lim _{m \rightarrow 1} \operatorname{cn}(t, m)=\operatorname{sech} t, \lim _{m \rightarrow 1} \operatorname{dn}(t, m)=\operatorname{sech} t .
\end{gathered}
$$

In view of (6) -(7) we may define $\operatorname{sn}(t, 0)=\sin t, \operatorname{cn}(t, 0)=\cos t, \operatorname{dn}(t, 0)=1$ and $\operatorname{sn}(t, 1)=\tanh t, \operatorname{cn}(t, 1)=\operatorname{sech} t, \operatorname{dn}(t, 0)=\operatorname{sech} t$. These functions are derivable and

$\frac{d}{d t} \operatorname{sn}(t, m)=\operatorname{cn}(t, m) \operatorname{dn}(t, m), \frac{d}{d t} \operatorname{cn}(t, m)=-\operatorname{sn}(t, m) \operatorname{dn}(t, m), \frac{d}{d t} \operatorname{dn}(t, m)=-m^{2} \operatorname{sn}(t, m) \operatorname{cn}(t, m)$. 


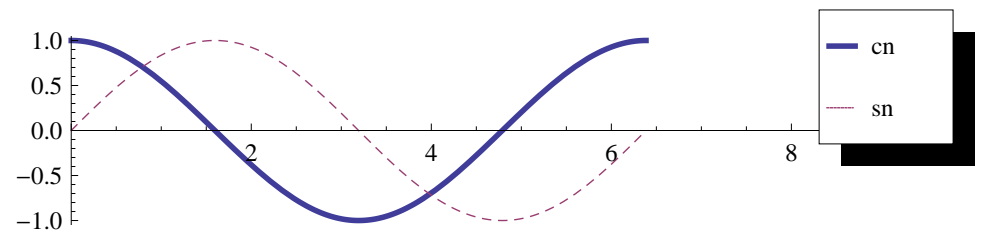

Figure 1: Graph of $\mathrm{sn}=\mathrm{sn}(t, 1 / 4)$ and $\mathrm{cn}=\mathrm{cn}(t, 1 / 4)$ on the interval $0 \leq t \leq$ 6.34 .

The graph of functions sn and cn are shown in Figure 1 for $m=\frac{1}{4}$.

From Figure 1 we see that functions sn and $\mathrm{cn}$ are periodic. They have a common period equal to $4 K(1 / 4)=4 K(\mathrm{~m})$, where $K=K(\mathrm{~m})$ is called the ellipticK function for modulus $m$. In our case, $K(1 / 4) \approx 1.5962422$.

Equations (6) say us that functions sn and cn generalize the sine and cosine functions respectively. In the linear case, the general solution to equation $x^{\prime \prime}(t)+\alpha x(t)=0$ is $x(t)=c_{1} \cos \left(\sqrt{\alpha} t+c_{2}\right)$, where $c_{1}$ and $c_{2}$ are the constants of integration which are determined from the initial conditions $x(0)=x_{0}$ and $x^{\prime}(0)=x_{0}^{\prime}$. When a cubic term is added we obtain the nonlinear equation $x^{\prime \prime}(t)+\alpha x(t)+\beta x^{3}(t)=0$ and the solution cannot be expressed in terms of cosine function. In this case the Jacobi cn funtion solves this nonlinear equation. Indeed, direct calculations using equations (8) show that function $x=c_{1} \operatorname{cn}\left(\omega t+c_{2}, m\right)$ satisfies following differential equation

$$
x^{\prime \prime}(t)+\omega^{2}\left(1-2 m^{2}\right) x(t)+\frac{2 m^{2} \omega^{2}}{c_{1}^{2}} x^{3}(t)=0
$$

for any constants $c_{1}$ and $c_{2}$. Therefore, the general solution to equation (2) is obtained by solving the system

$$
\alpha=\omega^{2}\left(1-2 m^{2}\right), \beta=\frac{2 m^{2} \omega^{2}}{c_{1}^{2}}
$$

which gives

$$
\omega=\sqrt{\alpha+c_{1}^{2} \beta} \text { and } m=\sqrt{\frac{c_{1}^{2} \beta}{2\left(\alpha+c_{1}^{2} \beta\right)}} .
$$

We have proved that the solution to initial value problem

$$
\ddot{x}+\alpha x+\beta x^{3}=0, x(0)=x_{0} \text { and } \dot{x}(0)=x_{0}^{\prime}(\beta \neq 0) .
$$

is

$$
x(t)=c_{1} \mathrm{cn}\left(\sqrt{\alpha+c_{1}^{2} \beta} t+c_{2}, \sqrt{\frac{c_{1}^{2} \beta}{2\left(\alpha+c_{1}^{2} \beta\right)}}\right)
$$


The values of $c_{1}$ and $c_{2}$ are determined from the initial conditions $x(0)=x_{0}$ and $\dot{x}(0)=x_{0}^{\prime}$. In order to find $c_{1}$ and $c_{2}$ we must solve following system :

$c_{1} \operatorname{cn}\left(c_{2}, m\right)=x_{0}$ and $-\sqrt{\alpha+c_{1}^{2} \beta} \operatorname{sn}\left(c_{2}, m\right) \operatorname{dn}\left(c_{2}, m\right)=x_{0}^{\prime}, m=\sqrt{\frac{c_{1}^{2} \beta}{2\left(\alpha+c_{1}^{2} \beta\right)}}$.

If $\dot{x}(0)=x_{0}^{\prime}=0$ then $c_{1}=x_{0}$ and $c_{2}=0$. In this case, the solution to initial value problem

$$
\ddot{x}+\alpha x+\beta x^{3}=0, x(0)=x_{0} \text { and } \dot{x}(0)=0 .
$$

is

$$
x(t)=x_{0} \operatorname{cn}\left(\sqrt{\alpha+\beta x_{0}^{2}} t, \sqrt{\frac{\beta x_{0}^{2}}{2\left(\alpha+\beta x_{0}^{2}\right)}}\right), \alpha+\beta x_{0}^{2} \neq 0 .
$$

Example 1 (for (16)). Let $\alpha=\beta=2, x_{0}=1$. Then the solution to problem $\ddot{x}+2 x+2 x^{3}=0, x(0)=1$ and $\dot{x}(0)=0$ is $x(t)=\mathrm{cn}(2 t, 1 / 2)$. This solution is bounded and periodic with period $2 K(1 / 2) \approx 3.3715$. See Fig. 2 .

Let $w=\alpha+\beta x_{0}^{2}=\omega^{2}$ and $\mu=\frac{\beta x_{0}^{2}}{2\left(\alpha+\beta x_{0}^{2}\right)}=m^{2}$. Then $x(t)=x_{0}$ $\operatorname{cn}(\sqrt{w} t, \sqrt{\mu})$. In the cases when $w<0$ or $\mu<0$ solution (16) may be written as follows [3] :

$$
x(t)=x_{0} \operatorname{nc}(\sqrt{-w} t, \sqrt{1-\mu}), w<0 \text { and } 0<\mu \leq 1 .
$$

Example 2 (for (17) with $0<\mu<1$ ). Let $\alpha=\beta=-2, x_{0}=1$. Then the solution to problem $\ddot{x}-2 x-2 x^{3}=0, x(0)=1$ and $\dot{x}(0)=0$ is $x(t)=\mathrm{cn}(2 \sqrt{-1} t, 1 / 2)=\mathrm{nc}(2 t, \sqrt{3} / 2)$. This solution is unbounded and periodic with period $2 K(\sqrt{3} / 2) \approx 4.313$. See Fig. 3 .

Example 3 (for (17) with $\mu=1$ ). Let $\alpha=1, \beta=-2, x_{0}=1$. Then the solution to problem $\ddot{x}+x-2 x^{3}=0, x(0)=1$ and $\dot{x}(0)=0$ is $x(t)=\mathrm{cn}(\sqrt{-1} t, 1)=\mathrm{nc}(t, 1)=\sec t$. This solution is unbounded and periodic with period $2 \pi \approx 3.31416$. See Fig. 4 .

$$
x(t)=x_{0} \operatorname{dc}\left(\sqrt{-w \mu} t, \sqrt{1-\frac{1}{\mu}}\right), w<0 \text { and } \mu>1 .
$$

Example 4 (for (18)). Let $\alpha=3, \beta=-1, x_{0}=2$. Then the solution to problem $\ddot{x}+3 x-x^{3}=0, x(0)=2$ and $\dot{x}(0)=0$ is $x(t)=$ $2 \mathrm{cn}(\sqrt{-1} t, \sqrt{2})=2 \mathrm{dc}(\sqrt{2} t, \sqrt{2} / 2)$. This solution is unbounded and periodic with period $4 K(\sqrt{2} / 2) / \sqrt{2} \approx 5.2441$. See Fig. 5 .

$$
x(t)=x_{0} \operatorname{cd}\left(\sqrt{w(1-\mu)} t, \frac{\sqrt{-\mu}}{\sqrt{1-\mu}}\right), w>0 \text { and } \mu<0 .
$$




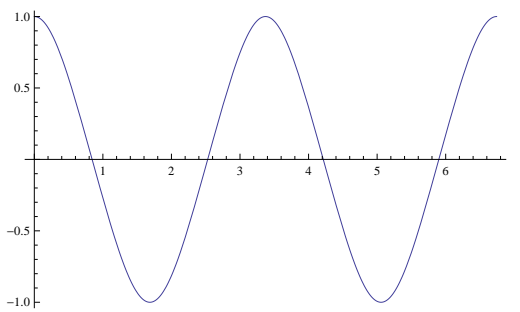

Figure 2: $x(t)=\mathrm{cn}(2 t, 1 / 2)$.

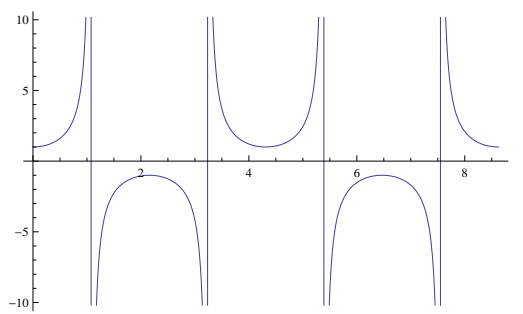

Figure 3: $x(t)=\mathrm{nc}(2 t, \sqrt{3} / 2)$.

Example 5 (for $(19)$ ). Let $\alpha=2, \beta=-1, x_{0}=1$. Then the solution to problem $\ddot{x}+2 x-x^{3}=0, x(0)=1$ and $\dot{x}(0)=0$ is $x(t)=\operatorname{cn}(t, \sqrt{-1} / \sqrt{2})=\operatorname{cd}(\sqrt{3 / 2} t, \sqrt{3} / 3)$. This solution is bounded with period $4 K(\sqrt{3} / 3) / \sqrt{2} \approx 8.0086$. See Fig. 6 .

$$
x(t)=x_{0} \operatorname{nd}\left(\sqrt{-w(1-\mu)} t, \frac{1}{\sqrt{1-\mu}}\right), w<0 \text { and } \mu<0 .
$$

Example 6 (for $(20)$ ). Let $\alpha=-2, \beta=13, x_{0}=10^{-2}$. Then the solution to problem $\ddot{x}-2 x+13 x^{3}=0, x(0)=10^{-2}$ and $\dot{x}(0)=0$ is $x(t)=$ $0.01 \mathrm{cn}(\sqrt{-1} 1.4142 t, \sqrt{-1} 0.018)=0.01 \mathrm{nd}(1.41398 t, 0.999837)$. This solution is bounded and periodic with period 15.2826. See Fig. 7.

We have obtained the solution to problem (15) in the form $x(t)=x_{0} \varphi\left(\omega_{0} t, m_{0}\right)$ in terms of Jacobi elliptic functions $\mathrm{cn}$, nc, dc, cd and nd having positive

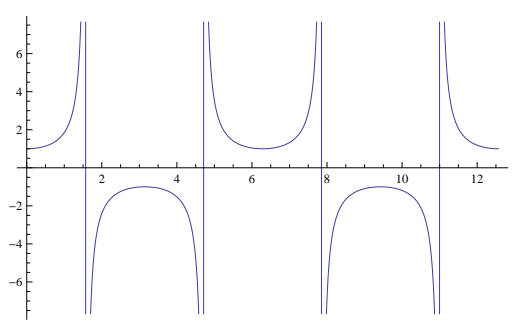

Figure 4: $x(t)=\sec t$. 


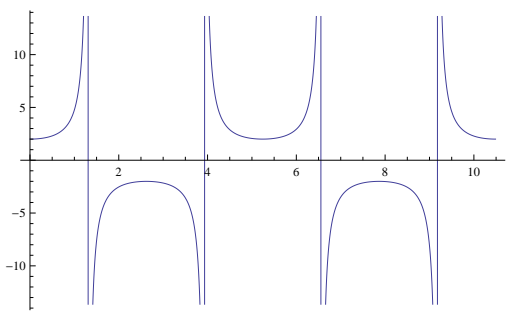

Figure 5: $x(t)=2 \operatorname{dc}(\sqrt{2} t, \sqrt{2} / 2)$.

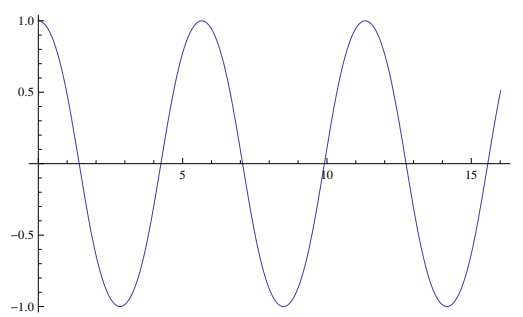

Figure 6: $x(t)=\mathrm{cd}(\sqrt{3 / 2} t, \sqrt{3} / 3)$.

frequency $\omega_{0}$ and modulus $m_{0}$ on the interval $[0,1]$ for all $\alpha, \beta$ and $x_{0}$ such that $\alpha+\beta x_{0}^{2} \neq 0$. It is clear from (17)-(18)-(19) and (20) that solution (16) to problem (15) is always real valued.

The behavior of solution (16) depends on the parameters $\alpha$ and $\beta$ and initial the condition $x_{0}$. Solution (16) is periodic and bounded if $\omega^{2}=w=$ $\alpha+\beta x_{0}^{2}>0$ for any $\beta$. Indeed, if $\beta>0$ then since $|c n(\omega t, m)|<1$ for any $m>0$ and $\omega>0$ then $|x(t)| \leq\left|x_{0}\right|$. See Example 1 and Fig. 2. On the other hand, if $\beta<0$ then since $\left|\operatorname{cd}\left(\omega_{0} t, m_{0}\right)\right|$, it follows from (19) that $|x(t)| \leq\left|x_{0}\right|$, where $\omega_{0}=\sqrt{w(1-\mu)}>0$ and $0<m_{0}=\frac{\sqrt{-\mu}}{\sqrt{1-\mu}}<1, \mu<0$. See Example 5 and Fig. 6. In the case when $\alpha+\beta x_{0}^{2}<0$ we obtain a bounded solution if $\beta>0$. See Example 6 and Fig. 7. If $\alpha+\beta x_{0}^{2}<0$ and $\beta<0$ the solution is

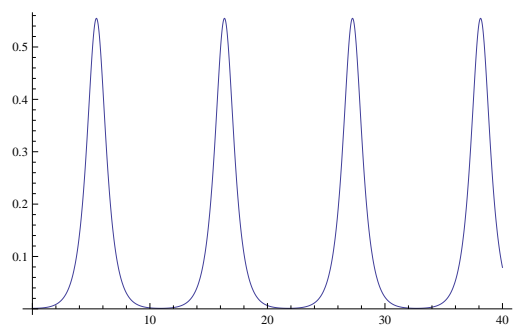

Figure 7: 
unbounded. See Examples 2, 3 and 4 and Figures 3, 4 and 5, respectively. If $\beta \rightarrow-\frac{2 \alpha}{x_{0}^{2}}$ then $m=\frac{\beta x_{0}}{2\left(\alpha+\beta x_{0}^{2}\right)} \rightarrow 1$ and since $\lim _{m \rightarrow 1} \operatorname{cn}(\omega t, m)=\operatorname{sech}(\omega t)$ solution (16) reduces to

$$
x(t)=x_{0} \operatorname{sech}(\sqrt{\alpha} t)
$$

Observe that solution (21) is bounded and non periodic. This situation is typical for solitons. Some important nonlinear differential equations admit soliton solutions tha are expressible in terms of sech.

On the other hand, if $\beta \rightarrow 0$ and $\alpha>0$ then $m=\frac{\beta x_{0}^{2}}{2\left(\alpha+\beta x_{0}^{2}\right)} \rightarrow 0$ and since $\lim _{m \rightarrow 0} \operatorname{cn}(\omega t, m)=\cos (\omega t)(16)$ degenerates into

$$
x(t)=x_{0} \cos (\sqrt{\alpha} t)
$$

which is the solution to linear equation $y^{\prime \prime}(t)+\alpha y(t)=0$. This result is consistent with the theory we know for the linear case.

\section{An Application : The Mathematical Pen- dulum.}

The pendulum is the archetypal dynamical system studied in nonlinear dynamics. Its nonlinear characteristics were studied by Rayleigh, and more-or-less the whole of Duffing's book was devoted to it.

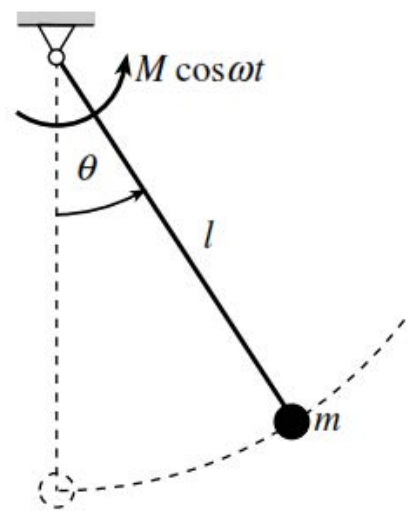

Figure 8: Mathematical Pendulum (picture taken from [2])

The equation of motion describing the angular displacement $\theta$ of the harmonically excited pendulum shown in Figure 8 is given by

$$
m l^{2} \frac{d^{2} \theta}{d t^{2}}+m g l \sin \theta=M \cos \omega t
$$


where $l$ is the length of the pendulum, $g$ is the acceleration due to gravity, and $M$ is the amplitude of the applied moment [2]. It can be seen that the pendulum has a softening stiffness characteristic because of the negative cubic term in the stiffness moment.

We are going to solve equation (22) for $M=0$, that is $m l^{2} \frac{d^{2} \theta}{d t^{2}}+m g l \sin \theta=0$. This equation may be written in the form

$$
\frac{d^{2} \theta}{d t^{2}}+\lambda^{2} \sin \theta=0, \theta(0)=\theta_{0}\left(0 \leq \theta_{0} \leq \pi\right), \theta^{\prime}(0)=0, \lambda=\sqrt{\frac{g}{l}}>0 .
$$

Direct calculations using (8) show that the solution to problem (23) is given by $\theta=\theta(t)=2 \arcsin (x(t))$, where $x(t)$ is the solution to initial value problem (15) with $x_{0}=\sin \left(\theta_{0} / 2\right), \alpha=\left(1+x_{0}^{2}\right) \lambda^{2}$ and $\beta=-2 \lambda^{2}$. Then, from (16) and (19) with $w=\lambda^{2} \cos ^{2}\left(\theta_{0} / 2\right)>0$ and $\mu=-\tan ^{2}\left(\theta_{0} / 2\right)<0$, we see that the solution to (23) may be written in the form

$$
\theta(t)=2 \arcsin \left[\sin \left(\frac{\theta_{0}}{2}\right) \operatorname{cd}\left(\sqrt{\frac{g}{l}} t, \sin \left(\frac{\theta_{0}}{2}\right)\right)\right],
$$

where $\operatorname{cd}(u, m)=\operatorname{cn}(u, m) / \operatorname{dn}(u, m)$.

Example 7. Let $x_{0}=1, \theta_{0}=60^{\circ}, g=9.8$ and $l=1 / 5$. Then the solution to problem $\frac{d^{2} \theta}{d t^{2}}+49 \sin \theta=0, \theta(0)=60^{0}=\pi / 3, \theta^{\prime}(0)=0$ is $\theta(t)=$ $2 \arcsin \left(\frac{1}{2} \operatorname{cd}\left(7 t, \frac{1}{2}\right)\right)$. Its period is $4 K(1 / 2) / 7 \approx 0.96$. Figure 9 shows the graph of this solution on $0 \leq t \leq 8 K(1 / 2) / 7$.

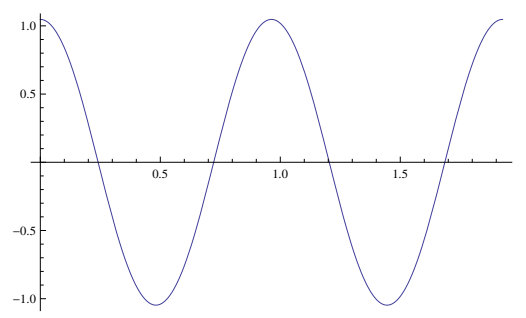

Figure 9: Graph of solution $\theta(t)=2 \arcsin \left(\frac{1}{2} \operatorname{cd}\left(7 t, \frac{1}{2}\right)\right)$.

\section{Conclusions}

We have derived the solution to both Duffing $\ddot{x}+\alpha x+\beta x^{3}=0(\beta \neq 0)$ for $\alpha+\beta x_{0}^{2} \neq 0$ and pendulum equations. Duffing equation is important in the study of nonlinear phenomena and nonlinear oscillations [1]. More details about Duffing equation may be found in [2]. A clear exposition about elliptic functions and their applications may be consulted in [4]. Some nonlinear partial differential equations admit solutions in terms of Jacobi elliptic functions 
[5]-[6].

\section{References}

[1] A.N. Nayfeth, D.T. Mook, Non-linear oscillations, John Wiley,New York, 1973.

[2] I. Kovacic, M. J. Brennan, The Duffing Equation: Nonlinear Oscillators and their Behaviour, Wiley, 2011. http://dx.doi.org/10.1002/9780470977859

[3] P.F. Bird, M. D. Friedman, Handbook of elliptic integrals for engineers and scientists, Springer, 1971. http://dx.doi.org/10.1007/978-3-642-65138-0

[4] J. V. Armitage, W. F. Eberlein, Elliptic Functions, Cambridge University Press, 2006. http://dx.doi.org/10.1017/cbo9780511617867

[5] A. H. Salas, J.E. Castillo H., New exact solutions to sinh-cosh-Gordon equation by using techniques based on projective Riccati equations, Computers \& Mathematics with Applications, Volume 61, Issue 2, January 2011, Pages 470-481. http://dx.doi.org/10.1016/j.camwa.2010.11.027

[6] A. H. Salas, Solving Nonlinear Partial Differential Equations by the snns Method, Abstract and Applied Analysis, Volume 2012 (2012), Article ID 340824. http://dx.doi.org/10.1155/2012/340824

Received: April 15, 2014; Published: December 8, 2014 